\title{
ON THE NUMBER OF IRREDUCIBLE MODULAR REPRESENTATIONS OF A FINITE GROUP IRVING REINER ${ }^{1}$
}

1. Let $K$ be a field of characteristic $p$, and let $G$ be a finite group. Denote by $n$ the L.C.M. of the orders of the $p$-regular elements of $G$, and let $\delta$ be a primitive $n$th root of 1 over $K$. Each $K$-automorphism of $K(\delta)$ is determined by a map $\delta \rightarrow \delta^{t}$ for some integer $t$, taken modulo $n$. The multiplicative group $T$ of all such exponents $t(\bmod n)$ is isomorphic to the Galois group of $K(\delta)$ over $K$.

Two $p$-regular elements $a, b \in G$ are called $K$-conjugate if $b^{t}=x^{-1} a x$ for some $x \in G$ and some $t \in T$. This defines an equivalence relation, relative to which the $p$-regular elements of $G$ are partitioned into $p$ regular $K$-conjugacy classes.

The following is due to Berman [2].

THEOREM. The number of irreducible $K$-representations of $G$ equals the number of p-regular $K$-conjugacy classes of $G$.

The purpose of this note is to present a simplified proof of Berman's theorem by making systematic use of Brauer characters. Let $\omega$ denote a primitive $n$th root of 1 over the rational field $Q$. To each $K$-representation $F$ of $G$ corresponds a Brauer character $\phi$, defined on the $p$-regular elements of $G$, as follows: For $p$-regular $a \in G$, let the characteristic roots of $F(a)$ be $\delta^{m_{1}}, \cdots, \delta^{m_{q}}$, and set

$$
\phi(a)=\omega^{m 1}+\cdots+\omega^{m q} \text {. }
$$

We refer the reader to [4] for the basic properties of Brauer characters, as well as for the other results used below.

Now let $\lambda_{1}, \cdots, \lambda_{k}$ be the Brauer characters of a full set of irreducible representations of $G$ in the algebraic closure of $K$. Then $\lambda_{1}, \cdots, \lambda_{k}$ are linearly independent over $Q(\omega)$, that is, if $\alpha_{1}, \cdots, \alpha_{k}$ $\in Q(\omega)$ are such that $\sum \alpha_{i} \lambda_{i}(g)=0$ for all $p$-regular $g \in G$, then each $\alpha_{i}=0$.

Finally, let $U$ be a $K$-representation of a subgroup $H$ of $G$, with Brauer character $\psi$. Then the Brauer character $\psi^{\theta}$ of the induced $K$ representation $U^{G}$ of $G$ is given by

$$
\psi^{G}(x)=[G: H]^{-1} \sum_{\boldsymbol{y} \in G} \psi\left(y^{-1} x y\right), \quad x \in G,
$$

Received by the editors April 21, 1963.

1 This research was supported by the Guggenheim Foundation and the Office of Naval Research. 
where $\psi$ coincides with $\psi$ on $H$ and vanishes outside of $H$. (See [3, \$25].)

2. Our proof of Berman's theorem depends on several straightforward lemmas.

LEMMA 1. Let $\phi_{1}, \cdots, \phi_{s}$ be the Brauer characters of a full set of irreducible $K$-representations $F_{1}, \cdots, F_{8}$ of $G$. Then $\phi_{1}, \cdots, \phi_{s}$ are linearly independent over $Q(\omega)$.

Proof. By $[4,(70.24)]$, the field $K(\delta)$ is a splitting field for $G$, and so each $\phi_{i}$ is a sum (with non-negative integral coefficients) of the Brauer characters $\lambda_{1}, \cdots, \lambda_{k}$ introduced above. From $[4,(29.6)$ and (69.4) ] it follows that for $i \neq j, \phi_{i}$ and $\phi_{j}$ have no summands in common. Since the $\lambda$ 's are linearly independent over $Q(\omega)$, so are the $\phi$ 's. This completes the proof.

LEMMA 2. Let $\phi$ be the Brauer character of a K-representation $F$ of $G$. If $a, b \in G$ are $K$-conjugate p-regular elements, then $\phi(a)=\phi(b)$.

Proof. Let $b^{t}=x^{-1} a x, x \in G, t \in T$. Then

$$
F(x)^{-1} F(a) F(x)=(F(b))^{t},
$$

so that $F(a)$ and $(F(b))^{t}$ have the same characteristic roots. But the characteristic roots of $(F(b))^{t}$ are the $t$ th powers of those of $F(b)$. Since each characteristic root of $F(b)$ is a power of $\delta$, and since $\delta \rightarrow \delta^{t}$ is an automorphism of $K(\delta)$ which leaves $F(b)$ unchanged, we conclude that $F(b)$ and $(F(b))^{t}$ have the same characteristic roots. The lemma is thus established.

Lemma 3. Let $a \in G$ be a p-regular element of $G$ of order $m$, and let $[a]$ denote the cyclic group it generates. Define $\theta$ by

$$
\theta\left(a^{t}\right)= \begin{cases}m, & t \in T, \\ 0, & t \in T .\end{cases}
$$

Then $\theta$ is a linear combination, with coeffcients from $Q(\omega)$, of Brauer characters of $K$-representations of $[a]$.

Proof. (Identical with the first paragraph of the proof of (42.5) in [4]. See also Berman [1], [2].)

3. We now prove Berman's theorem. Let $a_{1}, \cdots, a_{r}$ be representatives of the $p$-regular $K$-conjugacy classes in $G$, and let $\phi_{1}, \cdots$, $\phi_{s}$ be the Brauer characters of a full set of irreducible $K$-representations of $G$. By Lemma 2, each $\phi_{i}$ is completely determined by the $r$ tuple $\left(\phi_{i}\left(a_{1}\right), \cdots, \phi_{i}\left(a_{r}\right)\right)$. By Lemma 1 , these $s r$-tuples correspond- 
ing to $\phi_{1}, \cdots, \phi_{s}$, are linearly independent over $Q(\omega)$. Therefore $s \leqq r$.

On the other hand, for each $a_{i}$ we construct the function $\theta_{i}$ defined as in Lemma 3. Then $\theta_{1}^{G}, \cdots, \theta_{r}^{G}$ are linear combinations of $\phi_{1}, \cdots$, $\phi_{s}$ with coefficients from $Q(\omega)$. If we show that $\theta_{1}^{G}, \cdots, \theta_{r}^{G}$ are linearly independent over $Q(\omega)$, it will follow that $r \leqq s$, and we will be finished.

Let us compute $\theta_{i}^{\theta}\left(a_{j}\right)$. We have

$$
\theta_{i}^{G}\left(a_{j}\right)=[G: H]^{-1} \sum_{y \in G} \dot{\theta}_{i}\left(y^{-1} a_{j} y\right) .
$$

When $j \neq i$, this gives $\theta_{i}^{G}\left(a_{j}\right)=0$; for if $y^{-1} a_{j} y=a_{i}^{t}$ for some $y \in G$ and some $t \in T$, then $a_{i}$ and $a_{j}$ would be $K$-conjugate. On the other hand, in the expression for $\theta_{i}^{G}\left(a_{i}\right)$ the nonzero term $\dot{\theta}_{i}\left(a_{i}\right)$ always occurs, so that $\theta_{i}^{G}\left(a_{i}\right) \neq 0$. This completes the proof of the theorem.

We remark that the above proof is equally valid for $p=0$.

\section{REFERENCES}

1. S. D. Berman, p-adic ring of characters, Dokl. Akad. Nauk SSSR 106 (1956), 583-586.

2. - The number of irreducible representations of a finite group over an arbitrary field, Dokl. Akad. Nauk SSSR 106 (1956), 767-769.

3. R. Brauer and C. Nesbitt, On the modular characters of groups, Ann. of Math. 42 (1941), 556-590.

4. C. W. Curtis and I. Reiner, Representation theory of finite groups and associative algebras, Wiley, New York, 1962.

UNIVERSITY OF ILLINOIS 